\title{
Cutaneous Leishmaniasis as Imitator of Skin Diseases and a Diagnostic Challenge
}

\author{
Khalifa E. Sharquie 1,2*, Adil A. Noaimi'1,2, Banaz A. Saleh ${ }^{3}$ \\ ${ }^{1}$ Department of Dermatology, College of Medicine, University of Baghdad, Baghdad, Iraq \\ ${ }^{2}$ Iraqi and Arab Board for Dermatology \& Venereology, College of Medicine, Baghdad Teaching Hospital, Baghdad, Iraq \\ ${ }^{3}$ Center of Dermatology, Baghdad Teaching Hospital, Baghdad, Iraq \\ Email: *ksharquie@ymail.com, ksharquie@ymail.com, adilnoaimi@yahoo.com, banazali2014@yahoo.com
}

How to cite this paper: Sharquie, K.E., Noaimi, A.A. and Saleh, B.A. (2018) Cutaneous Leishmaniasis as Imitator of Skin Diseases and a Diagnostic Challenge. Journal of Cosmetics, Dermatological Sciences and Applications, 8, 158-177.

https://doi.org/10.4236/jcdsa.2018.83018

Received: December 24, 2017

Accepted: September 23, 2018

Published: September 26, 2018

Copyright $\odot 2018$ by authors and Scientific Research Publishing Inc. This work is licensed under the Creative Commons Attribution International License (CC BY 4.0).

http://creativecommons.org/licenses/by/4.0/

\begin{abstract}
Background: Cutaneous leishmaniasis (CL) usually has a well clinical presentation that rarely confused with other dermatological diseases, but when it presents with atypical picture, it may be a diagnostic challenge to dermatologist. Objective: To record the cases of CL those have very unusual presentations or sites in the Iraqi patients that might be confused with other dermatoses. Patient and methods: This is a clinical descriptive study that has been performed in the outpatient of Department of Dermatology Baghdad Teaching Hospital, Baghdad, Iraq, during the period from January 2015-February 2017, where fifty six patients were enrolled in the study. The age of the patients ranged from $1-67$ years old with median is 30 years, 32 males and 24 females with M:F ratio is $1.3: 1$, the diagnosis was confirmed by skin smear and/or biopsy. Results: Fifty six cases of CL with atypical presentations and unusual sites that categorized as follow: diffuse nodular 4 cases, paronychia like 1 case, sausage-like CL 4 cases, lupus vulgaris like 2 cases, rosacea like 1case, squamous cell carcinoma-like 3 cases, keratoacanthoma like 1 case, pyoderma gangrenosum-like 2 cases, erysipeloid like 1 case, B-cell lymphoma like 1 case, grouping pattern 1 case, panniculitis-like 2 cases, dermatitis like 2 cases, ecthyma like 1 cases, verruca like 1 case, keloid like 1 case, CL on the scalp 1 case, palmer CL 1 case, CL on the eyelid 1 case, CL on the ear 1 case, $\mathrm{CL}$ on the lips 3 cases, one genital CL case and twenty cases of sporotrichosis like spread. Conclusion: Unusual presentations of the CL with atypical lesions should be well recognized and investigated to confirm the diagnosis so that not to be confused with other skin diseases especially in countries where cutaneous leishmaniasis is not endemic disease.
\end{abstract}

\section{Keywords}

Cutaneous Leishmaniasis, Atypical Presentation, Diagnostic Challenge 


\section{Introduction}

The clinical feature of Cutaneous Leishmaniasis (CL) tends to vary between and within regions, reflecting a different species of parasite, the type of zoonotic cycle, immunological status and also perhaps due to genetically determined response of the patients. The clinicians should be aware of the wide varieties of the clinical presentations possibilities. The Old World Cutaneous Leishmaniasis (OW-CL) is caused by five species of Leishmania: L. major, L. tropica, L. aithiopica and to L. donovoni infantum [1].

Iraq is an endemic area since ancients and recently cutaneous leishmaniasis is running a severe outbreak due to wars and terrorism where hundreds of cases had been seen in last three years. The most species that have been isolated by polymerase chain reaction technique are L. major (60\%) and L. tropica that responsible on the majority of the cases [2].

The morphological spectrum of OW-CL are wide, but the main "classical" types are due to $L$. major also known as wet, rural or zoonotic CL, after a short incubation period of less than 4 months, multiple (up to 100 insect bite-like lesions on the exposed body part), if not treated, spontaneously healing within 2 8 months leaving large atrophic disfiguring scars. While CL due to L. tropica also known as dry, urban or anthroponotic CL, has a long incubation period about 8 months, and produce single or multiple lesions that heal spontaneously within a period twice as long as moist type leading to disfiguring scars or progress to chronic CL or L. recidevans [3] and even these two variants could not be easily recognized by the clinical pictures.

The histopathological changes of CL includes: epidermal hyperplasia with ortho/parakeratosis, while in some cases the epidermis shows necrosis and atrophy, and some sections show pseudoepitheliomatous hyperplasia, follicular plugging and liquefaction degeneration of the basal layer, lymphocytic exocytosis is also recorded in many patients. While the dermal changes are mainly observed as a spectrum, where in the early ulcerative lesion, there are dense and diffuse mixed inflammatory cells mainly lymphocyte, histiocyte and plasma cell infiltration. These plasma cells are present in foci and when they are plenty, encourage diagnosing cutaneous leishmaniasis. While in the dry and chronic lesions, the dermal changes are mainly granuloma with few lymphocytes and plasma cells. The hallmark of the disease (observed in $70 \%$ of the cases) is the presence of numerous extracellular and intracellular (within histiocyte) amastigotes also known as Leishman-Donovan (LD) bodies. In Iraq (Sharquie communication), the presence of $\mathrm{LD}$ bodies in the tissue was seen in $30 \%$ of cases and was observed by both Hematoxylin and Eosin stain as well as by Giemsa stains. More recently, both sepal and lobular panniculitis appear to be as an important histopathological finding in the cutaneous lesions, and can differentiate CL from other skin lesions that might simulate it clinically like chronic skin lesion, discoid lupus erythematous and cutaneous lymphoma [4] [5].

The classical types usually diagnosed easily by the dermatologist especially in 
the endemic areas, but in some instances the patients present with an unusual presentation that are not familiar to the physician. These presentation have been reported in the literature and designated as "atypical" cases because they are infrequent, and these cases given different names according to: the lesion's configuration like (annular, chancriform, keloid, acneiform, panniculitis, verrucous), its similarities to the other diseases like (lupoid, eczematous, erysipeloid, sporotrichoid, localized lymphadenitis, psoriasiform, rhinophymatous, mycetomatous, zosteriform, cheilitis) or to the anatomical localization of the lesion like (palmoplantar, genital, paronychial, perioral, periorbital land scalp) [6]-[19].

These cases unless suspected, they might mismanaged and running in a chronic non-diagnosed course.

The aim of present descriptive study is to record many cases of CL that had a strange clinical presentation, in order to help other dermatologist worldwide to do not misdiagnosed these cases that lead to mismanagement.

\section{Patients and Methods}

Fifty six patients with different unusual cutaneous leishmaniasis presentations were enrolled in this descriptive study that performed in the outpatient of Department of Dermatology Baghdad Teaching Hospital, Baghdad during January 2015-February 2017, with their ages range from 1 - 67 years old with the median 30 years and 32 males and 24 females with M/F ratio 1.3:1. All these cases diagnosed clinically and confirmed by smear and/or histopathological study after high suspicion of CL. Regarding treatment of these cases, for mild and single lesion, topical $25 \%$ podophyllin in tincture benzoin solution is used, while for more diffuse and disseminated cases oral ketoconazole in combination with oral zinc sulfate are used [20] [21].

\section{Results}

\subsection{Diffuse Nodular CL}

Four cases of diffuse nodules

- Forty five years old female with history of right side mastectomy she was on chemotherapy due breast carcinoma, presented with multiple ulcerated and crusted papules mainly on the right upper extremity for 6 months durations and the diagnosis approved by demonstrated Leishman donovan bodies (LD) in the histopathological study (Figures 1(a)-1(c)).

- Diffuse truncal nodular CL: Thirty five years old healthy male patient presented with diffuse nodule on the trunk for more than one year (Figure 2(a) and Figure 2(b)).

- Diffuse facial nodular CL: four years old girl presented with diffuse crusted papules on the forehead and cheeks for 2 months durations (Figure 3).

- Post-kala azar dermal leishmaniasis (PDKL): Six years old boy has history of kala azar when he was four years old, presented with history of small papules begun around his mouth with small hypo-pigmented macules on the 
abdomen diagnosed as post-kala azar dermal leishmaniasis. After few months the lesions became diffuse as small plaques on the face and nodular lesions on the trunk. (Figure 4(a) and Figure 4(b))



(a)

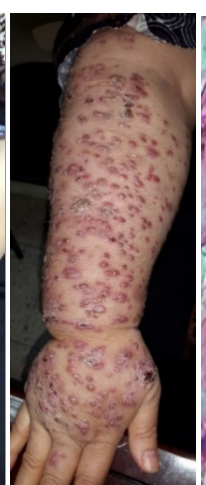

(b)

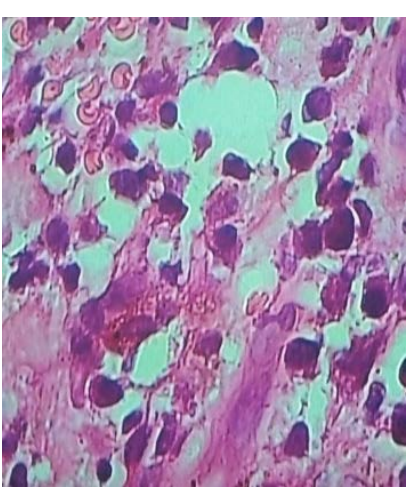

(c)

Figure 1. DiffuseNodular CL. (a) \& (b) 45 years old female with multiple ulcerated and crusted papules mainly on the right arm for 6 months durations diagnosis approved by demonstrating Leishman donovan bodies (LD) inside the histiocyte cells in the histopathology (c).

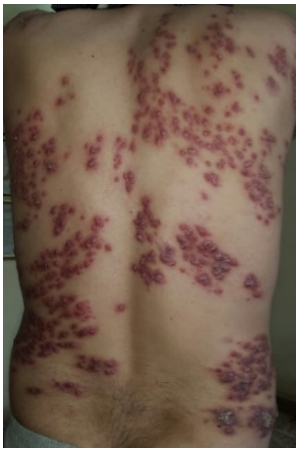

(a)

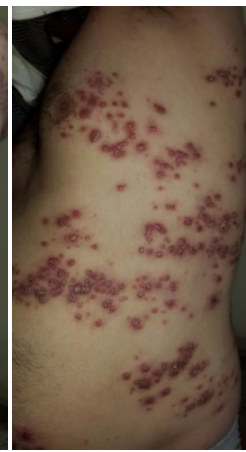

(b)



(c)

Figure 2. Diffuse truncal CL (a) (b). 35 years old presented with diffuse nodule on the trunk for more than one year. (c) Skin H/P of the patient showed intra cellular LD bodies inside histiocyte cells.

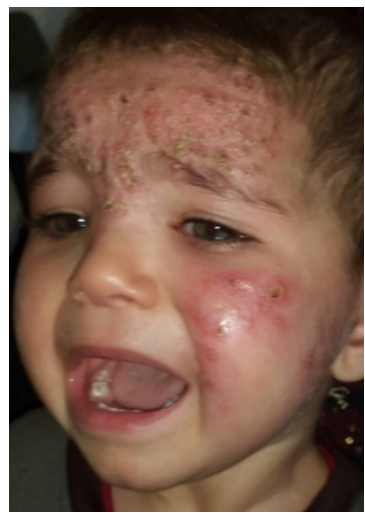

Figure 3. Diffuse facial CL. 4 year's old girl presented with diffuse papules on the forehead and cheeks for 2 months durations. 


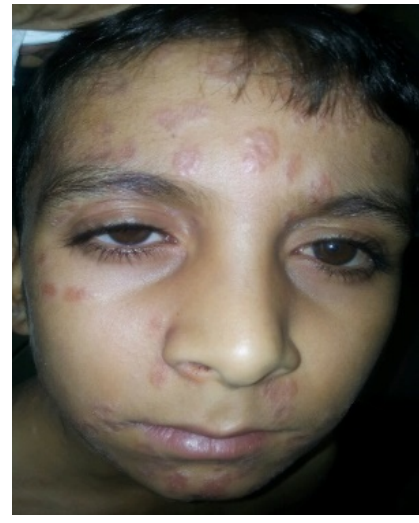

(a)

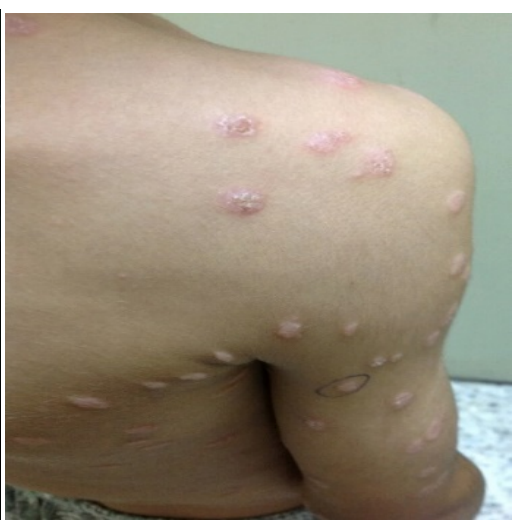

(b)

Figure 4. Post kala azar dermal leishmaniasis: A small plaques on the face (b) wide spread nodular lesions on the trunk of the same patient.

\subsection{Paronychia Like CL}

Thirty one years old male presented with redness and painless swelling in the right index finger for more than one month duration (Figure 5).

\subsection{Sausage-Like CL}

Four cases of sausage-like lesions on the fingers and toes:

- Thirty four years old female patient with sausage-like CL of middle finger of the right hand for two months duration (Figure 6).

- Twenty nine years old female with sausage-like CL of the ring finger of the left hand for 5 months duration.

- Sixty two years old male presented with multiple sausage-like CL of both hands for more than 5 months duration.

- Thirty three years old male patient presented with painless erythematous swelling in the second toe of the left foot for two months duration (Figure 7).

\subsection{Lupus Vulgaris Like}

Two cases with lupus vulgaris like:

- Eighteen years old dwarfism patient presented with history of facial lesion for one year diagnosed as cutaneous tuberculosis and received anti TB for 6 months without improvement and biopsy was done and LD bodies were obvious in the histopathological study. The patient received systemic pentavalent antimony treatment with excellent response (Figure 8).

- Sixty seven years old male patient present with history of facial lesion for 3 months duration (Figure 9).

\subsection{Rosacea Like}

Fifty five years old female patient with erythematous skin lesions on the right cheek diagnosed and treated wrongly as asymmetrical rosacea for more than 7 months duration (Figure 10). 


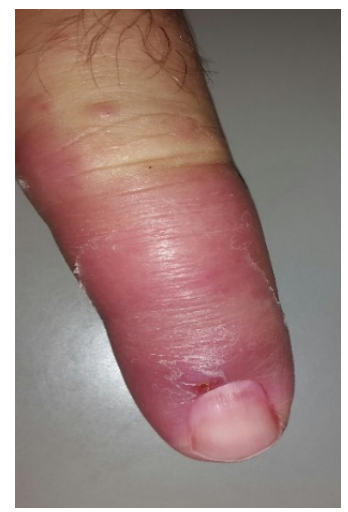

Figure 5. Paronychia like CL. 31 years old male presented with redness and painless swelling in the right index finger for more than one month duration.

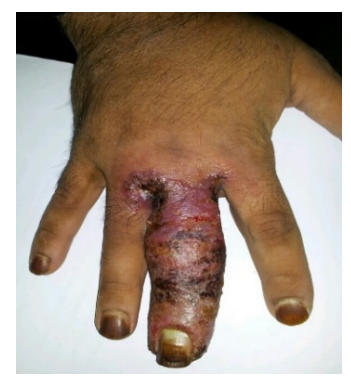

Figure 6. Sausage-like CL in the finger. Thirty four old female patient with sausage-like CL of middle finger of the right hand for two months duration.

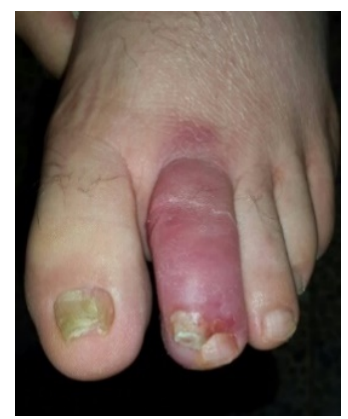

Figure 7. Sausage like CL in the $2^{\text {nd }}$ toe. Thirty three years old male patient presented with painless erythematous swelling of the second toe of the left foot for two months duration.

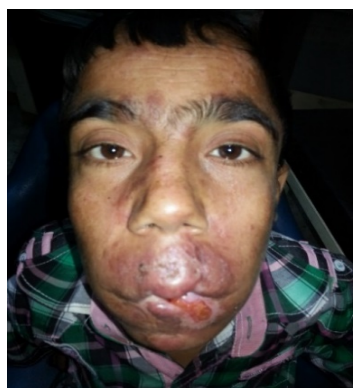

Figure 8. Lupus vulgaris like: 18 years old dwarfism patient presented with history of facial lesion for more than 6 months. 


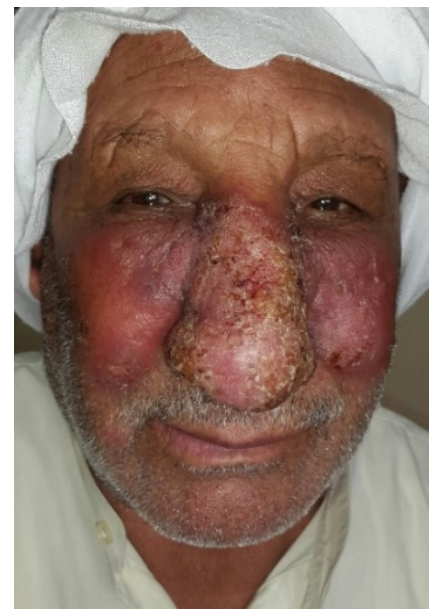

Figure 9. Lupus vulgaris like. 67 years old male patient present with history of facial lesion for 3 months duration.

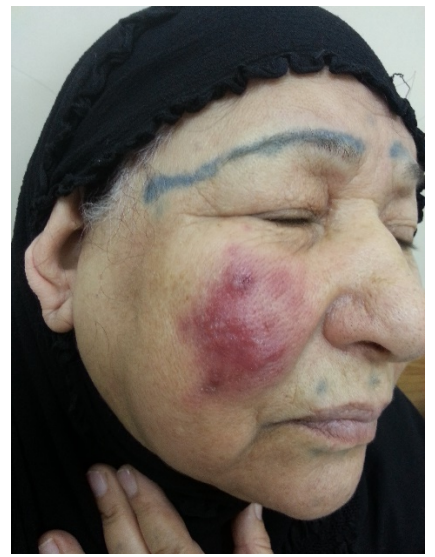

Figure 10. Rosacea like. 55 years old female patient with erythematous skin lesions on the right cheek diagnosed and treated wrongly as rosacea for more than 7 months duration.

\subsection{Squamous Cell Carcinoma (SSC) Like-CL}

Three cases with SCC-like lesions:

- Thirty five years old male patient presented with history of large ulcer lesion on the right elbow for 2 months duration (Figure 11).

- Forty four years old female with large fungating mass on her nose for more than 15 years duration with multiple history of surgical removal and recurrence, biopsy reveled granuloma with obvious LD in the field (Figures 12(a)-12(c)).

- Thirty years old male present with large non healing ulcer on the left hand for 3 months duration.

\subsection{Keratoacanthoma Like-CL}

Fifty five years old male presented with skin lesion that has typical crater of kertoacanthoma on the right hand for 3 months duration (Figure 13). 


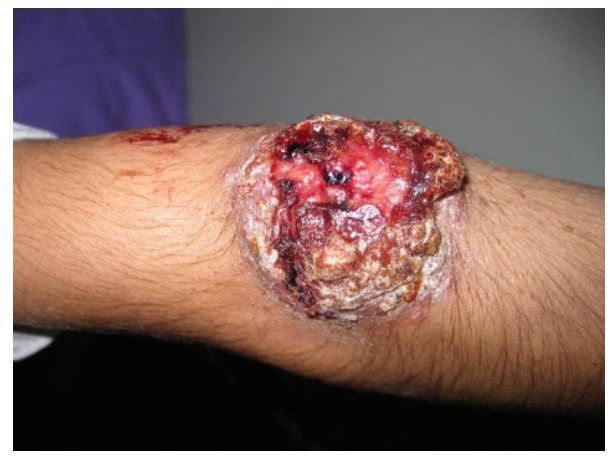

Figure 11. Squamous cell carcinoma like. 35 years old male patient presented with history of large ulcer lesion on the right elbow for 2 months duration.

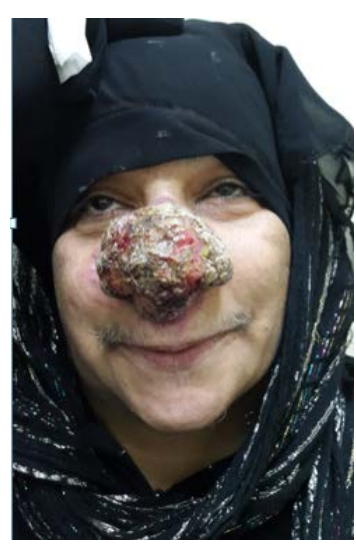

(a)

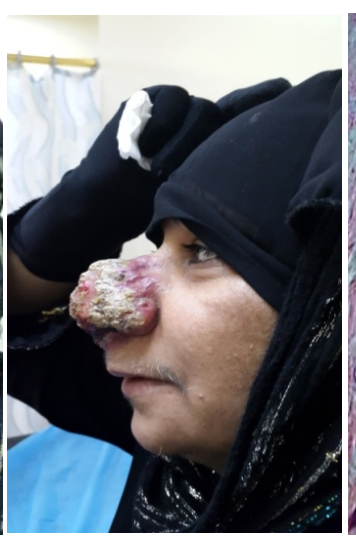

(b)

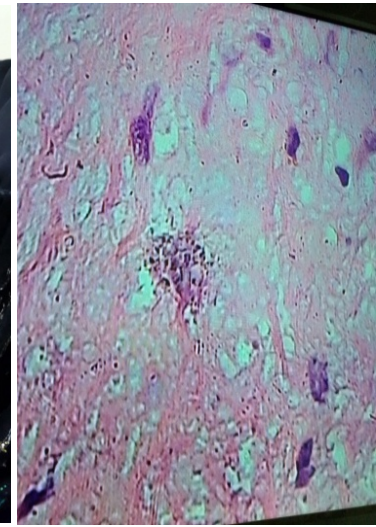

(c)

Figure 12. (a) (b) SCC like CL, 44 years old female with large fungating mass on her nose for more than 15 years duration. (c) H/P of the patient showed intra and extra cellular LD bodies inside histiocyte cells.

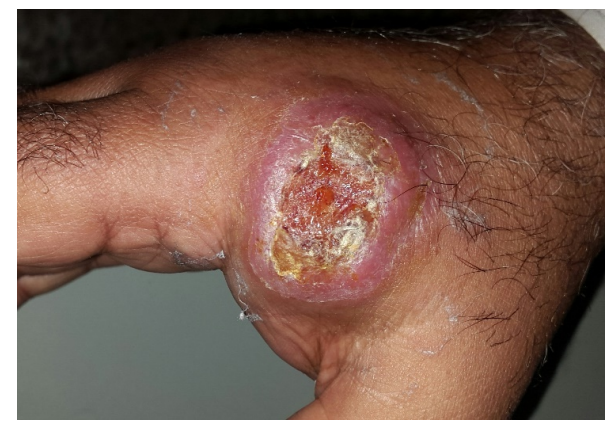

Figure 13. Keratoacanthoma like. 53 years old male seen with skin lesion that have typical crater on the right hand for 3 months duration.

\subsection{Pyoderma Gangrenosum Like CL}

Thirty years old healthy female presented with single large undermined edge ulcerative lesion on the right lower leg for 3 months duration (Figure 14(a) and Figure 14(b)).

Twelve years old male presented with large ulcer on the post, ankle joint for 2 months duration. 


\subsection{Erysipeloid Like CL}

Fifty sixth years old female with localized erythematous plaque on the right cheek for 5 month duration (Figure 15).

\subsection{B Cell Lymphoma Like CL}

Sixty one years old female patient presented with diffuse crusted plaque of the face for 6 months durations (Figure 16(a) and Figure 16(b)).

\subsection{Grouping Pattern CL}

Thirty years old male patient presented with multiple grouped nodules on the right arm for 2 months duration (Figure 17).

\subsection{Panniculitis Like CL}

Two cases of painful subcutaneous nodule with redness of overlying skin.

- Twenty nine years old female presented with tender subcutaneous nodule on the right leg for two months duration (Figure 18).

- Thirty four years old male had history of the typical CL on the dorsum of the right foot associated with subcutaneous tender with overlying skin redness of the anterior right shin.

\subsection{Dermatitis Like CL}

Two cases with dermatitis like:

- Eighteen years old male patients with discoid eczema like on the right foot for 2 months duration (Figure 19).

- Twenty three years old female presented with dermatitis like CL on the right hand for 3 months duration (Figure 20).

\subsection{Ecthyma Like CL}

one year boy with 2 skin lesion with crusted lesion on the right cheek for 1 month duration and another lesion on the lower lip (Figure 21).

\subsection{Verruca Like CL}

Nineteen years old male patient presented with multiple warty plaques on the right leg for 7 months duration (Figure 22).

\subsection{Keloid Like CL}

Twenty two years old male patient with painless smooth hard lesion on the lower right eyelid for more than 3 month duration (Figure 23).

\subsection{CL on the Scalp}

Forty one year's male presented with ulcerated plaque on the scalp for 3 months duration, as it is unlikely to acquire CL on the scalp but it might be due to non-hairy scalp (Figure 24). 




(a)

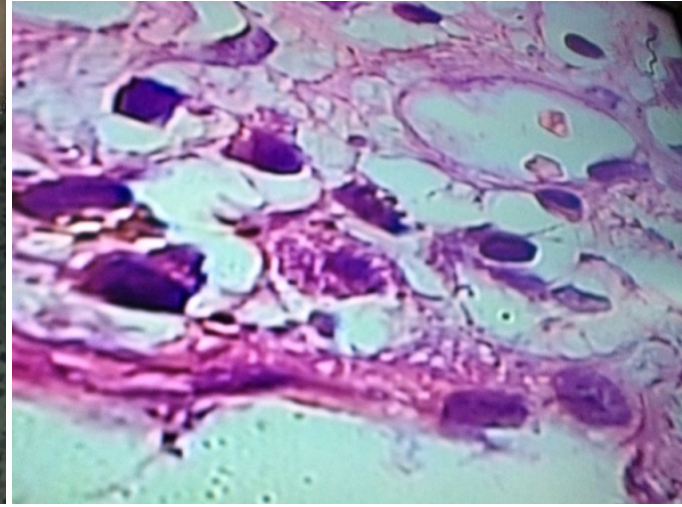

(b)

Figure 14. Pyoderma gangrenosum like CL. 30 years old healthy female presented with single large undermined edge ulcerative lesion on the right lower leg for 3 months duration. $\mathrm{C} \mathrm{H} / \mathrm{P}$ showed intracellular LD bodies inside histiocyte cells.

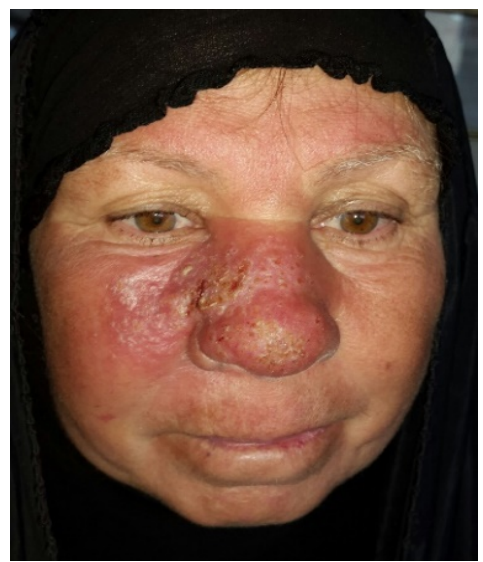

Figure 15. Erysipeloid like CL. 56 years old female with localized erythematous plaque on the right cheek for 5 month duration.

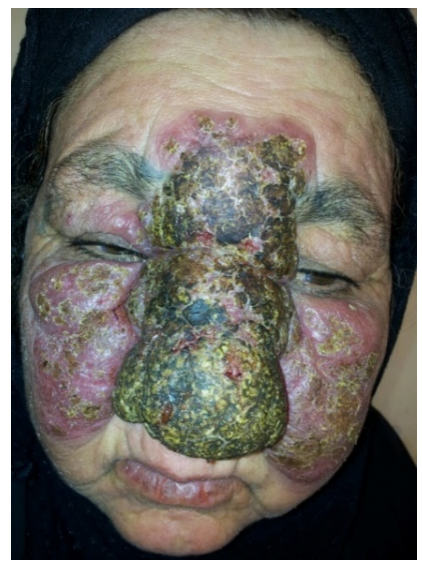

(a)

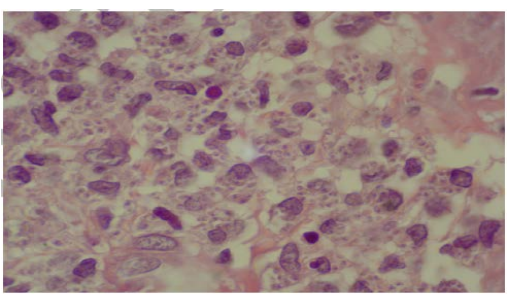

(b)

Figure 16. B cell lymphoma like CL. 61 years old female patient presented with diffuse crusted plaque of the face for 6 months durations. B H/P showed numerous intracellular L.D bodies inside histiocytes. 


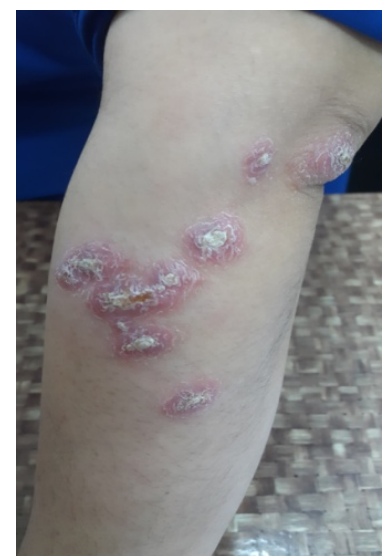

Figure 17. Grouping pattern CL. 30 yers old male patient presented with multiple grouped nodules on his right arm for 2 months duration.

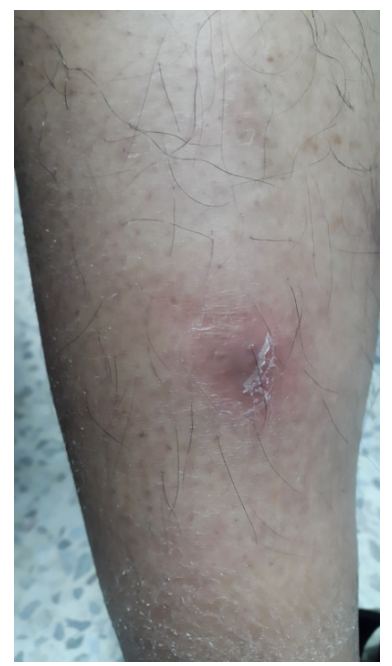

Figure 18. Panniculitis like CL. 29 years old female presented with tender subcutaneous nodule on the right leg for two months duration

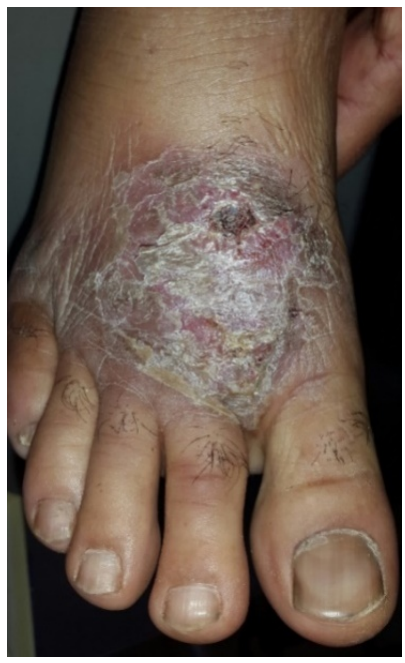

Figure 19. Discoid eczema like. 18 years old male patients with discoid eczema like on the right foot for 2 months duration. 


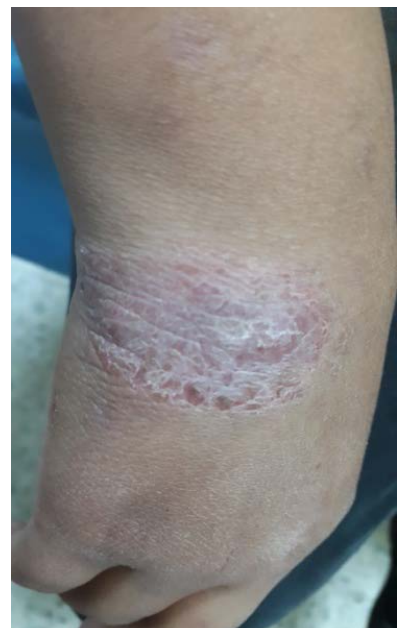

Figure 20. Dermatitis like CL. 23 years old female presented with dermatitis like CL on the right hand for 3 months duration.

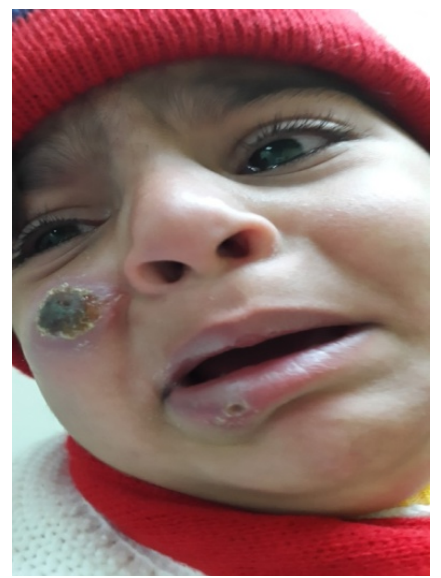

Figure 21. Ecthyma like. One year boy with 2 skin lesion with crusted lesion on the right cheek for 1 month duration and another lesion on the lower lip.

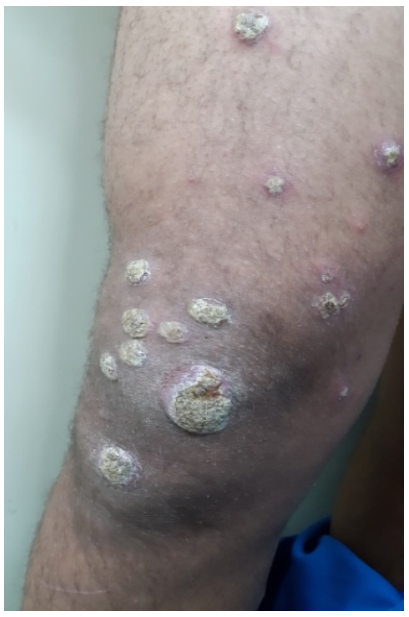

Figure 22. Verruca like CL. Nineteen years old male patient presented with multiple warty plaques on the right leg for 7 months duration. 


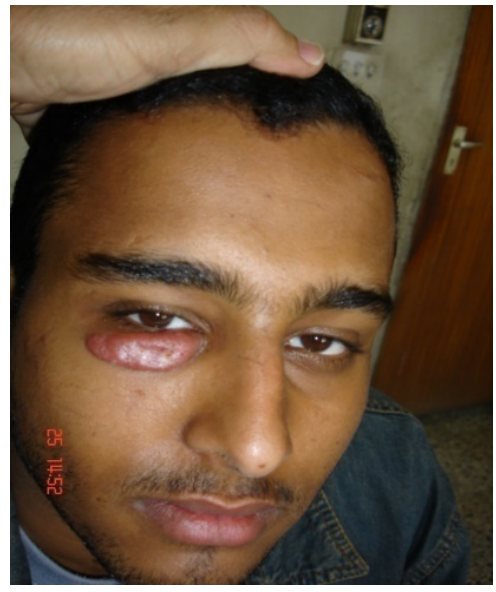

Figure 23. Keloid like. 22 years old male patient with painless smooth hard lesion on the lower right eyelidfor more than 3 month duration.

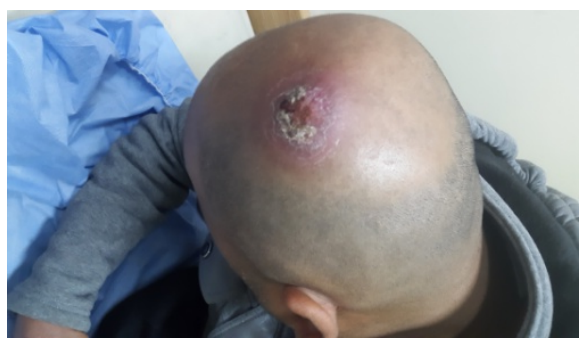

Figure 24. CL on the scalp. 41 year's old bald male presented with ulcerated plaque on the scalp for 3 months duration.

\subsection{Palmer CL}

Thirty six years old female presented with ulcerative plaque on the palmer surface of the right hand for 5 month duration (Figure 25).

\subsection{CL on the Eyelid}

Twelve month's girl with single crusted lesion on the upper right eyelid for 2 months duration (Figure 26).

\subsection{CL on the Ear}

Three years old girl with more than 5 crusted lesions on the left ear pinna for 2 months duration (Figure 27).

\subsection{CL on the Lip}

Three cases of CL on the lips:

- Twelve years old female with painless indurated and crusted lesion associated with lower lip swelling for 2 months duration (Figure 28).

- Twenty eight years old male with crusted lesion the lower lip for 1 month duration.

- Fourteen years old male with skin lesion on the upper lip for 1 month duration (Figure 29). 


\subsection{Genital CL}

Fifty eight years old male presented with two painless indurated crusted nodules on the scrotum with typical CL on his right hand (Figure 30 ).

\subsection{Sporotrichosis Like}

Twenty cases were seen, that commonly affecting the upper extremities, when the primary lesions of CL on the hands, the sporotrichosis like lesion along the lymphatics of forearms (Figure 31). This rash usually seen long time after the start of the primary lesion and appear as linear subcutaneous nodulation along the forearm and usually stop at elbow and sometime associated with lymphadenopathy of elbow region and rarely seen progressing above the elbow towards axilla, but when the primary CL on the elbow, the linear sporotrichoid rash passing into the axilla (Figure 32). In some cases it might superficial and appear as a linear erythematous nodules along the lymphatic. The sporotrichosis like rash was rarely seen on the legs, but occasionally seen on the face (Figure 33). This rash usually resolved after treatment of primary CL.

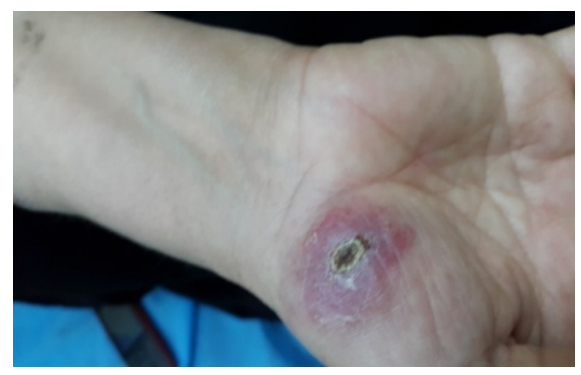

Figure 25. Palmer CL: thirty sixth years old female presented with ulcerative plaque on the palmer surface of the right hand for 5 month duration.

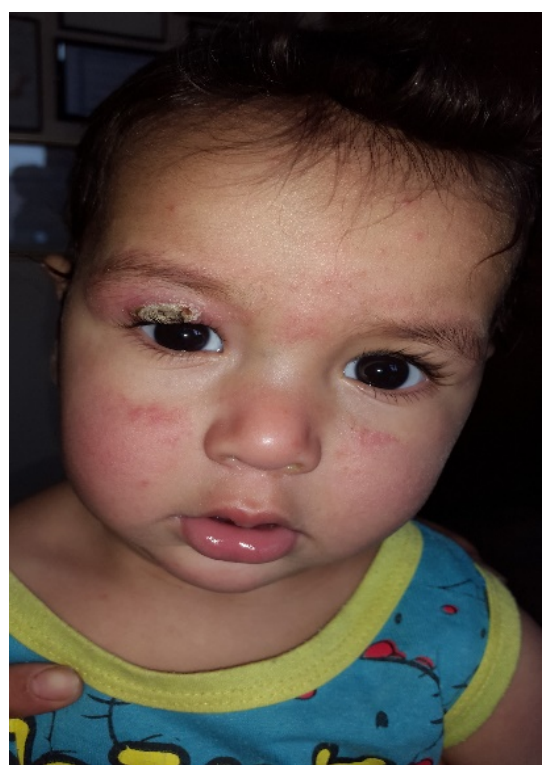

Figure 26. CL on the eyelid.Nine months girl with single crusted lesion on the upper right eyelid for 2 months duration. 


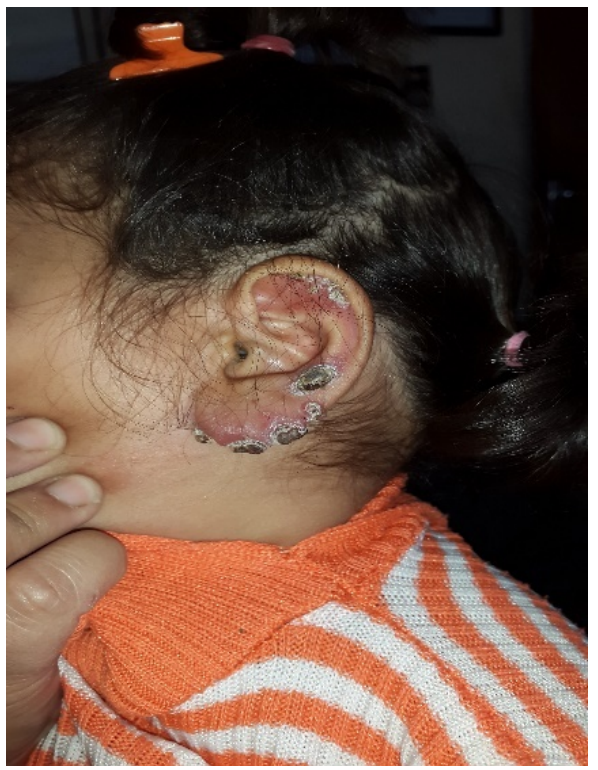

Figure 27. CL on the ear. Three years old girl with more than 5 crusted lesions on the left ear pinna for 2 months duration.

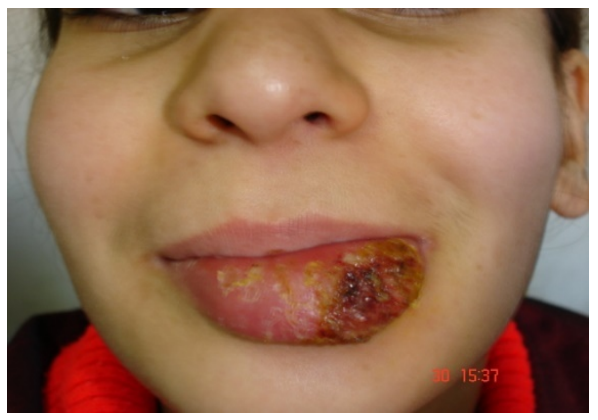

Figure 28. CL on the lower lip. Twelveyear's old female with indurated and crusted lesion associated with lower lip swelling for 2 months duration.

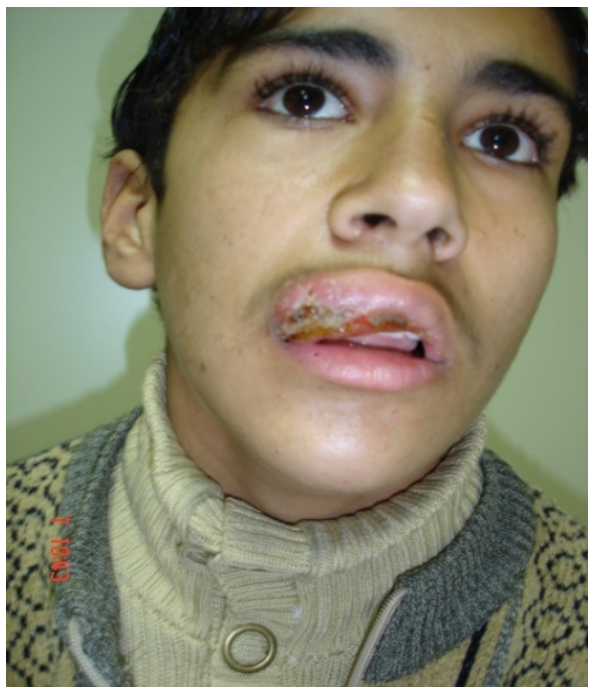

Figure 29. CL in the upper lip. 14 year's old male with skin lesion on the upper lip for 1 month duration. 


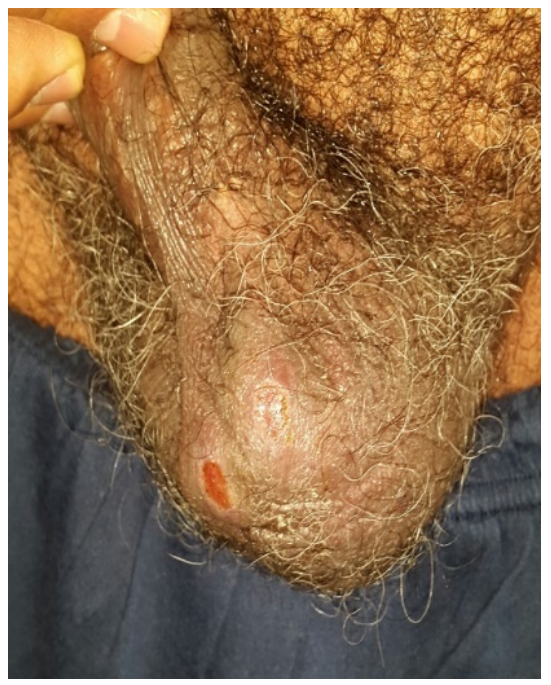

Figure 30. CL on the scrotum. A: 58 years old male presentedwith painless indurated crusted nodule.

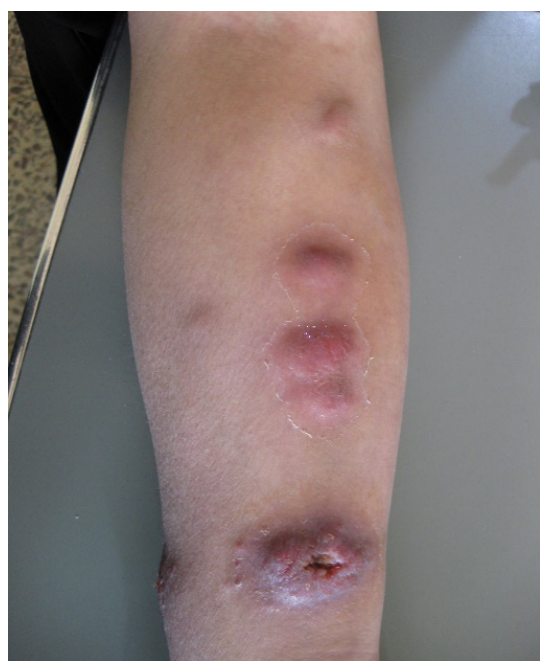

Figure 31. Sporotichosis like: The primary lesion of CL on the wrist and spirotochosis like along forearm stop at elbow.

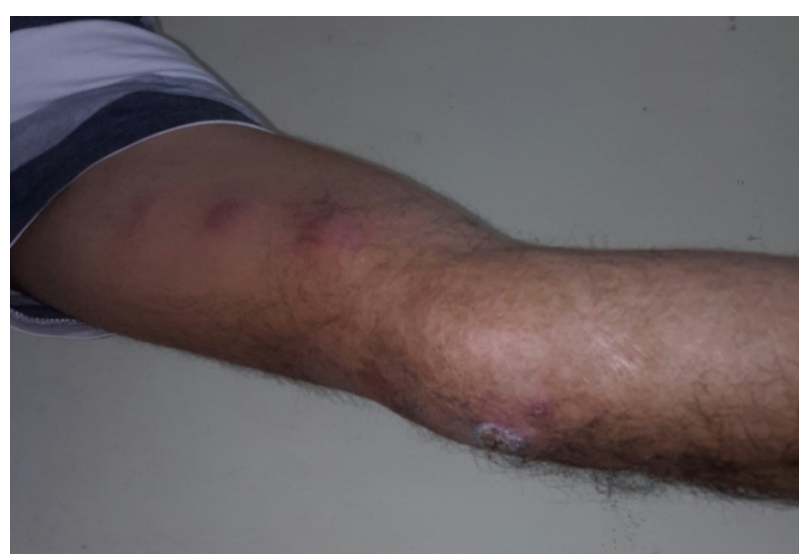

Figure 32. Sporotrichosis like: the primary lesion of CL on the elbow and the sporotrichosis like pass into axilla. 


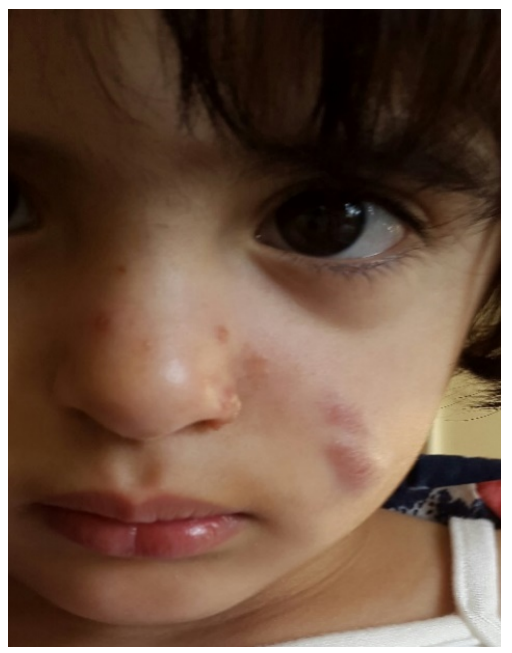

Figure 33. Sporotrichosis like on the face: the primary lesion on the left nostril and sporotrichosis along left cheek.

\section{Discussion}

Cutaneous leishmaniasis (CL) is a zoonotic disease that transmitted by bite of sand-fly that carries the parasite, and it has many presentations, depend on the species and host related factors like malnutrition, immunosuppression and resistance to the disease [2].

$\mathrm{CL}$ is a worldwide distribution and WHO considered it as category 1 disease (emerging and uncontrolled), and the cause of recent geographic spread is attributed to massive rural-urban migration, natural disasters, global warming, wars and deforestation. The most classification is mainly to region where infection is acquired into: Old World CL and New World CL and both subgroup differ regarding causative agent, vectors, clinical presentation and prognosis. In Old world $\mathrm{Cl}$, the most presentation are zoonotic (rural, moist) type and anthroponotic (urban, dry) type depend mainly on the exposed part of the body like face and extremities, however there are many other atypical presentation like: eczamatoid, psoraziform, paranochial, chanciform, annular, verrucous, and in atypical site like on palm, scalp, eyelid, genital area etc. The causes of the atypical presentation are unclear, however it is could be related to local immune response, skin temperature, microcirculation of the dermis where the parasite inoculated, and specific subtypes of parasite [22].

In Iraq and in the last few years there are obvious an increasing number of CL that was noticed especially among Iraqi immigrants due to their unhealthy and crowded environment where they forced to live because of war and terrorism [23].

In the present work, we reported many cases of unusual presentations and strange sites that need high index of suspicion because of endemicity of the disease in Iraq, although many of these presentations had been already described in literature, but many of them never described before, and there are high recommendation for further studies to explain the cause of these atypical presentation 
that could be due to changes in the serotypes of species. In addition, CL should be included in the differential diagnosis of many common and uncommon dermatological diseases in order to correct diagnosis and avoid misdiagnosis by other dermatologist especially in areas with less endemic of the disease that led to mismanagement and ending with disfiguring outcome or progression to chronic state.

The most interesting cases are: the diffuse nodular type, sausage like of fingers and toes, lupus vulgaris like of face, rosacea like, squamous cell carcinoma like especially of the face, keratoacanthoma like, pyoderma gangrenosum like, B-cell lymphoma like, grouping pattern that might simulate bed bug rash, discoid eczema like, warty like. Also lesions on unusual sites were seen like on the eyelid, mouth lips, scalp, scrotum, palms and ears. Sporitrichoid CL dissemination is an uncommon presentation, but it has been reported as atypical presentation and many reports of evoked after intra-lesional treatment of the primary lesion. It has been described in three pattern of spread: 1) subcutaneous nodules in proximity to the primary lesion, 2) dilated palpable lymphatic vessels in form of a "beaded cord" and 3) reginal lumphadenitis.It is more seen in New World CL, In Old World, the exact prevalence is unknown but ranges from 10\%-19\% in the previous records, and the majority of the reported cases were shown to be caused by $L$. major and sometime due to L. tropica [24]. In Iraq, the sporotrichosis due to deep fungal infection or to other causes are not reported, but in $\mathrm{CL}$, it is not a rare to see sporotrichoid lesions and present as a linear subcutaneous nodular lesions along the course of lymphatic although could superficial erythematous nodulations as seen in the present study. This rash resolves after treatment of the primary lesion.

These strange atypical morphological lesions and especially on the unusual sites like scalp and genitalia should published so dermatologists especially those working in non-endemic areas will be familiar with these rashes otherwise will be misdiagnosis and mismanagement.

\section{Conclusion}

Atypical presentations of CL or lesions on the unusual sites should be published, so dermatologists especially those working in non-endemic areas will be familiar with these cases otherwise they will be misdiagnosed and mismanaged.

\section{Conflicts of Interest}

The authors declare no conflicts of interest regarding the publication of this paper.

\section{References}

[1] World Health Organization (2010) Control of Leishmaniasis. Report of the WHO Expert Committee on the Control of Leishmaniasis, Geneva.

[2] Sharquie, K.E., Al-Najar, S.A., Noaimi, A.A. and Al-Heany, A.R. (2014) Cutanane- 
ous Leishmaniasis: Comparative Techniques for Diagnosis. Journal of Dental and Medical Science, 13, 33-37.

[3] Ghosn, S.H. and Malek, J.M. (2012) Infestations, Bites and Stings. In: Wolff, K., Goldsmith, L.A., Katz, S.I., Gilchrest, B.A., Paller, A.S. and Leffell, D.J., Eds., Fitzpatrick's Dermatology in General Medicine, 8th Edition, McGraw-Hill-Company, New Work, 2527-2537.

[4] Lever, W. and Schaumburg-Lever, G. (1983) Diseases Caused by Protozoa. In: Lever, W. and Schaumburg-Lever, G., Eds., Histopathology of the Skin, 6th Edition, JB Lippincott Co, Philadelphia, 356-359.

[5] Sharquie, K.E., Hameed, A.F. and Noaimi, A.A. (2016) Panniculitis Is a Common Unrecognized Histopathological Features of Cutaneous Leishmaniasis. Indian Journal of Pathology and Microbiology, 59, 16-19.

[6] Nelson, S.A. and Warschaw, K.E. (2012) Protozoa and Worms In: Bolognia, J.L., Jorrizo, J.L. and Schaffer, J.V., Eds., Dermatology, Elsevier, Amsterdam, 1391-1397.

[7] Veraldi, S. (2006) Psoriasiform Cutaneous Leishmaniasis. International Society of Dermatology, 45, 129-130. https://doi.org/10.1111/j.1365-4632.2006.02691.x

[8] Salmanpour, R., et al. (1999) Erysipeloid Leishmaniasis: An Unusual Clinical Presentation. European Journal of Dermatology, 9, 458-459.

[9] Robati, R.M. and Abdollahimajd, F. (2015) Cutaneous Leishaniasis: Reported of Two Atypical Cases. Journal of Clinical Medicine Researches, 2, 1-3.

[10] Raja, K.M. (1998) Unusual Clinical Variants of Cutaneous Leishmaniasis in Pakistan. British Journal of Dermatology, 139, 111-113. https://doi.org/10.1046/j.1365-2133.1998.02325.x

[11] Lahiry, A.K. (2002) Unusual Presentation of Cutaneous Leishmaniasis. Indian Journal of Dermatology, Venereology and Leprology, 68, 145-146.

[12] Azadeh, B. (1985) "Localized" Leishmania Lymphadenitis: A Light and Electron Microscopic Study. The American Journal of Tropical Medicine and Hygiene, 34, 447-455. https://doi.org/10.4269/ajtmh.1985.34.447

[13] Kubba, R. (1987) Dissemination in Cutaneous Leishmaniasis. I. Subcutaneous Nodules. International Journal of Dermatology, 26, 300-304. https://doi.org/10.1111/j.1365-4362.1987.tb00193.x

[14] Ul Bari, A. and Ber Rahman, S. (2008) Many Faces of Cutaneous Leishmaniasis. Indian Journal of Dermatology, Venereology and Leprology, 74, 23-27. https://doi.org/10.4103/0378-6323.38402

[15] Calvopina, M., Gómez, E.A., Uezato, H., Kato, H., Nonaka, S. and Hashiguchi, Y. (2005) Atypical Clinical Variants in New World Cutaneous Leishmaniasis: Disseminated, Erysipeloid, and Recidivans Due to Leishmania (V.) Panamensis. The American Journal of Tropical Medicine and Hygiene, 73, 281-284. https://doi.org/10.4269/ajtmh.2005.73.281

[16] Iftikhar, N., Bari, I. and Ejaz, J. (2003) Rare Variants of Cutaneous Leishmaniasis: Whitlow, Paronychia, and Sporotrichoid. International Journal of Dermatology, 42, 807-809. https://doi.org/10.1046/j.1365-4362.2003.02015.x

[17] Bari, A.U. (2012) Clinical Spectrum of Cutaneous Leishmaniasis: An Overview from Pakistan. Dermatology Online Journal, 18, 4.

[18] Omidian, M. and Mapar, M.A. (2006) Chronic Zosteriform Cutaneous Leishmaniasis. Indian Journal of Dermatology Venereology and Leprology, 72, 41-42. https://doi.org/10.4103/0378-6323.19717 
[19] Momeni, A.Z. and Aminjavaheri, M. (1994) Clinical Picture of Cutaneous Leishmaniasis in Isfahan, Iran. International Journal of Dermatology, 33, 260-265. https://doi.org/10.1111/j.1365-4362.1994.tb01039.x

[20] Sharquie, K.E., Noaimi, A.A. and AL-Ghazzi, A.G. (2015) Treatment of Cutaneous Leishmaniasis by Topical 25\% Podophyllin Solution (Single, Blinded, Therapeutic, Controlled Study). Journal of Dermatology \& Dermatologic Surgery, 19, 108-113.

[21] Sharquie, K.E., Noaimi, A.A. and Al-Salam, W.S. (2016) Treatment of Acute Cutaneous Leishmaniasis by Oral Zinc Sulfate and Oral Ketoconazole Singly and in Combination. Journal of Cosmetics, Dermatological Science and Applications, 6, 105-115. https://doi.org/10.4236/jcdsa.2016.63014

[22] Moralis, C.A. and Palacio, J. (2014) Zosteriform Cutaneous Leishmaniasis Due to Leishmania (Vinnia) Panamesis and Leishmanial (Vinnia) Brazilensis Report of Three Cases. Biomedica, 34, 340-344.

[23] AL-Timimi, T.H. (2015) Cutaneous Leishmanisis among Immigrants in Iraq. Journal of Biology, Agriculture and Healthcare, 21, 11-14.

[24] Thomaidou, E., Horev, L., Jotkowitz, D. and Zamir, M. (2015) Lymphatic Dissemination in Cutaneous Leishmaniasis Following Local Treatment. The American Journal of Tropical Medicine and Hygiene, 93, 770-773.

https://doi.org/10.4269/ajtmh.14-0787 